\title{
LA RESOLUCIÓN DE PROBLEMAS EN EL ÁREA DE BIOQUÍMICA: UN ENFOQUE COGNITIVO Y METACOGNITIVO
}

\author{
Dolffi Rodríguez Campos*, Pedro Nel Zapata Castañeda ${ }^{* *}$
}

\begin{abstract}
Abstrac
The following paper shows the results of an investigation of pre-experimental type about of the cognitives and metacognitives processes implicates in the problems resolution in Biochemistry ares. The investigation is carried out with a group of 12 students of the technological Corporation of Bogotá during the year 2001. The obtained results showed that the students of the population, previous to the application of the strategy, they did not had the cognitives abilities related to the problems definition, strategies selection and solution supervision. Through a training program in the different cognitives and metacognitives abilities is achievement in the population a notable improvement in the use of the mentioned abilities.
\end{abstract}

Key words: Ability, cognition, metacognition, solving problem, Biochemistry

\section{RESUMEN}

El siguiente articulo muestra los resultados de una investigación de tipo preexperimental, sobre los procesos cognitivos y metacognitivos implicados en la resolución de problemas, en el área de Bioquímica. La investigación se llevó a cabo con un grupo de 12 estudiantes de la corporación tecnológica de Bogota, durante el año 2001. Los resultados obtenidos, mostraron que los estudiantes de la población, previo a la aplicación de la estrategia, no tenían las habilidades cognitivas relacionadas con la definición de problemas, selección de estrategias y supervisión de soluciones. A través de un programa de intervención cognitivo en las distintas habilidades cognitivas y metacognitivas, se logró en la población una mejora notable en el uso de las habilidades mencionadas.

Palabras clave: habilidades, cognición, metacognición, resolución de problemas, bioquímica.

\section{Introducción}

La resolución de problemas ha sido un área de estudio no sólo en el campo de la psicología sino también, en el campo educativo. Se considera que la resolución de problemas como estrategia de enseñanza es importante para favorecer el desarrollo de habilidades de pensamiento y aproximar a los estudiantes a lo que es la actividad de los científicos. Adicionalmente, en el campo educativo, los profesores, principalmente de

\footnotetext{
* Magíster en Docencia de la Química U.P.N. Especialista en Análisis Químico instrumental. P.U.J. Profesora Universidad Javeriana.

** Profesor Departamento de Química. Universidad Pedagógica Nacional. Magíster en Docencia de la Química U.P.N. Candidato a Doctor Educación en Ciencias U.P.N. E-

mail:pzapata@ióuni.pedagogica.edu.co
} 
ciencias, atribuyen mucha importancia a la resolución de problemas, aunque realmente se trate de ejercicios, no sólo como estrategia de enseñanza, sino como una forma de evaluar el aprendizaje de sus estudiantes.

La resolución de problemas es una línea de investigación alrededor de la cual confluyen aportes de la epistemología, la ciencia cognitiva, la pedagogía y la didáctica, siendo éste último campo en donde se concretan, confrontan y validan muchos de los avances en estas áreas.

En el marco de la resolución de problemas, es evidente que la mayoría de los alumnos, incluso los profesores, no son conscientes de los procesos cognitivos y metacognitivos implicados en su resolución. Este aspecto trae como consecuencia que los profesores orienten la resolución de problemas hacia la aplicación de algoritmos que, por lo general, producen fijación funcional e impiden el uso de diversas habilidades intelectuales relacionadas con el pensamiento divergente y convergente.

La investigación aquí presentada sobre los procesos cognitivos y metacognitivos implicados en la resolución de problemas, se fundamenta en los avances conceptuales y metodológicos producidos en más de 50 años de investigación en este campo. Ciertamente, la bibliografía es extensa y múltiples son los estudios en éste campo, igualmente diversas son sus aplicaciones, no sólo en el ámbito de la sicología de carácter cognitivo, sino también en el educativo.

Por otro lado, aunque si bien la investigación en el área de la bioquímica es importante, también lo es la investigación en el campo de la enseñanza y el aprendizaje de esta disciplina que tengan en cuenta, entre otras cosas, no sólo el aprendizaje de los conocimientos disciplinares, sino también el desarrollo de las habilidades y destrezas propias del pensamiento científico.

\section{Marco referencial}

La mayor parte de los estudios sobre resolución de problemas se han enfocado por lo menos en tres grandes áreas: en la primera, se han estudiado los modos como las personas procesan la información para resolver problemas; en la segunda, se han estudiado las estrategias de resolución y, en la tercera, los conocimientos necesarios para resolverlos.

La mayor parte de las investigaciones sobre resolución de problemas en el campo de la didáctica de las ciencias experimentales, se han orientado a entrenar a los sujetos en protocolos de resolución, descuidando la importancia que tiene para el estudiante los procesos metacognitivos necesarios para la adecuada comprensión y solución de problemas.

En lo referente a ¿qué es un problema?, varios autores han planteado diversidad de definiciones. Perales (1993) afirma que "por problema puede entenderse cualquier situación prevista o espontánea que produce, por un lado, un cierto grado de incertidumbre y, por el otro, una conducta tendiente a la búsqueda de su solución" ( $p$. 170).

Gil y Martínez (1988) afirman que se puede considerar problema una 'situación que presenta dificultades para la cual no existen soluciones evidentes, pues una vez conocidas éstas dejan de constituir problemas" (p. 131). 
Por otra parte, Garret (1988), define el problema como "una situación enigmática"; es decir, "aquella que no es solucionable ni resoluble, sino sólo comprensible" (p. 225). Estas situaciones son denominadas por este autor "problemas verdaderos", mientras que las que pueden ser solucionadas dentro de un paradigma las denomina "rompecabezas". También plantea que "cada persona, en dependencia de su personalidad, de las estrategias o recursos de que disponga y de su conocimiento, puede tomar una determinada situación, bien como problema, bien como rompecabezas, lo que lleva a pensar que tomar una determinada situación como problema o no, es algo estrictamente personal" (p. 226).

En el caso de la presente investigación, se entiende que las situaciones problemáticas que se plantean a los estudiantes constituyen problemas para ellos en la medida en que no conocen de forma inmediata la solución, aunque estas situaciones ya tengan solución para los científicos o incluso el profesor, en la medida que éste ya conoce por lo menos la estrategia a seguir y la solución que se obtendrá. Así pues, las situaciones problemáticas planteadas a los estudiantes no constituyen problemas de frontera en el campo de una ciencia o disciplina particular, como la bioquímica, sino situaciones para las que los estudiantes, por lo menos, no conocen de forma evidente e inmediata la solución.

En este mismo contexto, la resolución de problemas ha sido entendida de diversas maneras. Así pues, autores como Novak (1988), Cárdenas (1998), Amnestoy (1998) y Kempa (1986), han planteado diversidad de conceptualizaciones y modelos acerca de la resolución de problemas. Para Kempa (1986) "la resolución de problemas constituye un proceso mediante el cual se elabora la información en el cerebro del sujeto que lo resuelve; dicho proceso requiere de la memoria a corto y largo plazo, e implica no solo la comprensión del problema, sino la selección y utilización de estrategias que le permitan llegar adecuadamente a su solución" (p. 99).

Por otra parte, Polyá (1982), considera que "en el campo de las matemáticas la resolución de problemas consiste tanto en un proceso de aprendizaje como en un objetivo en sí mismo, así como una técnica básica que debe ser desarrollada" (p. 126).

Jessup (1998), plantea algunos puntos esenciales a tener en cuenta para la resolución de problemas como parte de la enseñanza de las ciencias:

-Comprensión del área de conocimiento de la cual fue extraído el problema; es decir, la existencia de un dominio de conocimiento.

- El modelo de resolución deberá ayudar al alumno a plantear hipótesis, así como a diseñar e implementar estrategias o experimentos que le permitan corroborar o improbar dichas hipótesis.

- La comprobación de la solución constituye la fase final del proceso de solución.

- Los procesos seleccionados deberían ser tomados de una situación natural (p. 45).

Según esta autora "la resolución de problemas se refiere a procesos de conducta y pensamiento dirigidos hacia la ejecución de una determinada tarea intelectualmente exigente" (p. 46). 


\section{La metacognición}

Según Nickerson, Perkins y Smith (1994) el conocimiento metacognitivo es:

"el conocimiento sobre el metaconocimiento y el saber, e incluye el conocimiento de las capacidades y limitaciones de los procesos del pensamiento humano, de lo que se puede esperar que sepan los seres humanos en general y de las características de personas específicas -en especial de uno mismo- en cuanto a individuos conocedores y pensantes" (p. 125).

Estos autores también plantean que "las habilidades metacognitivas se pueden considerar como aquellas habilidades que son útiles o necesarias para la adquisición, el empleo y el control del conocimiento, y de las demás habilidades cognitivas. Estas habilidades cognitivas también incluyen la capacidad de planificar y regular el empleo eficaz de los propios recursos cognitivos.

Así pues, el metaconocimiento para estos autores se entiende como "una experiencia cognoscitiva que facilita la adquisición, el uso y el control del conocimiento". Además, consideran que "la sustancia del conocimiento se puede dividir o clasificar a través de tres tipos de variables: las variables personales, de la tarea y de la estrategia" (p. 125).

La primera variable "abarca todo lo referente al conocimiento acerca de que uno puede llegar a quedarse sin comprender algo por no llegar a conseguir una representación coherente de ello o conseguir una representación coherente pero incorrecta, esto podría considerarse como un ejemplo que encaja dentro de las variables personales" (p. 125).

La segunda categoría se refiere a las variables de la tarea y hacen referencia al conocimiento de lo que implican las características de una tarea cognitiva en cuanto a la dificultad de ésta y al mejor modo de enfocarla" (p. 125).

Por último, se tiene la categoría o variable de la estrategia que según estos mismos autores, implica el "conocimiento de los meritos relativos de los diferentes enfoques de una misma tarea cognitiva. La mayor parte del conocimiento metacognitivo implica probablemente la existencia de interacciones o combinaciones entre dos o tres tipos de variables" (p. 125).

Además, explican que la experiencia de la "sensación de saber" (o de la sensación de no saber), como también la sensación de saber que uno tiene (o no ) probabilidades de resolver un problema en el que está trabajando, puede calificarse de experiencia metacognitiva" (p. 125).

Las habilidades metacognitivas participan en los procesos del pensamiento, en este aspecto es en el que se han centrado muchos trabajos sobre metacognición, los cuales buscan que el individuo conozca sus capacidades al igual que sus limitaciones y que, además, aprenda a emplear las primeras y a evitar las segundas, con habilidad. Este proceso es de gran importancia en muchas profesiones y vocaciones en las que se requiere ser capaz de valorar su propio nivel de destreza.

Por otra parte, es necesario examinar la influencia del conocimiento en la solución de problemas en un ambito determinado. Miller (1962), señala que "aunque un conocimiento adecuado de la materia en cuestión es necesario para tener un desempeño idóneo, no constituye una garantía suficiente de lograrlo" (p. 37). Otro ejemplo, es el dado por Barrows y Tablyn (1980), quienes aseguran "que la posesión de un importante cuerpo de 
conocimiento en materia médica, por ejemplo, no garantiza que quien la posee sabrá cuándo y cómo aplicar ese conocimiento en el cuidado de los pacientes" (p. 38).

Lo anterior apunta a explicar que una persona no solo necesita tener una serie de conocimientos de un campo específico para un buen desempeño, sino que necesita tener el conocimiento de cómo y cuándo debe aplicarlo dentro de un contexto determinado; por lo tanto, se puede plantear que cualquier trabajo que se haga sobre el mejoramiento de las habilidades metacognitivas debe propender a convertir el sujeto en un usuario hábil del conocimiento.

En este mismo sentido, algunos ejemplos de habilidades metacognitivas identificados por varios autores, son la planificación, la predicción, la verificación, la comprobación de la realidad y la supervisión y control de los intentos deliberados de llevar a cabo tareas intelectualmente exigentes.

Las capacidades de evaluar tanto las aptitudes como las actitudes respecto a una tarea específica, la capacidad de controlar y evaluar el propio desempeño en esa tarea, son otros tipos de habilidades. Por ejemplo, cuando alguien estudia, llega un momento en que deja de hacerlo porque tiene confianza en que el trabajo que está realizando está lo suficientemente arraigado para ser reproducido.

Según Nickerson, Perkins y Smith (1994), "entre las habilidades que parecen intervenir en muchos casos de comprensión, están las que se necesitan para controlar esa comprensión, especialmente la capacidad de comprender que uno no entiende una parte de lo que ha oído o leído y el conocimiento de lo que tiene que hacer al respecto" (p. 130). La habilidad de conocer lo que uno sabe o no, es lo que en el mismo campo, diferencia un experto de un novato.

Cuando el individuo desarrolla habilidades metacognoscitivas se facilita la planificación de estrategias; además, el sujeto es consciente de la estrategia que está usando para resolver el problema y puede hacer supervisión de los logros alcanzados, entre otros.

En el desarrollo de este trabajo, específicamente, la temática se restringió a los procesos cognitivos y metacognitivos implicados en la resolución de problemas. Fundamentalmente, el trabajo se sustentó en la teoría triarquica de la inteligencia de Stenberg (1990), en lo referente a la subteoría componencial que define los procesos directivos para el uso de información, planificación y el control de soluciones.

Según Amnestoy (1998):

"En la resolución de problemas, las dificultades que se observan no están solo relacionadas con el
procesamiento de los datos para encontrar la solución, sino también con una etapa anterior que tiene
relación con la definición de una estrategia que especifique los pasos para alcanzar la meta deseada, los
procedimientos para verificar los logros y para propiciar la retroalimentación necesaria. Para tal fin es
necesario el uso del metaconocimiento, el cual se refiere al conocimiento consciente acerca del
conocimiento, es la habilidad para saber qué sabe y qué ignora, y sus potencialidades o limitaciones, el
grado de dificultad o de complejidad de una situación problema; la trascendencia de sus actos, etc. Con
relación a esto "los metacomponentes son precisamente los procesos directivos, previos a la acción que
permiten definir cómo llevar a cabo y controlar cualquier actividad antes de actuar. Dichos procesos se
identifican como: la planificación, la supervisión y el control" (p. 33).

Según esta misma autora: 
Los procesos directivos se subdividen en los siguientes subprocesos. Definición del problema, definición de la estrategia para resolver el problema, representación mental del problema, distribución de recursos y supervisión de soluciones. Las facultades para aplicar estos procesos constituyen las cinco habilidades metacognoscitivas que permiten aplicar los procesos directivos. Cada uno de los procesos contribuye a alcanzar una etapa de la planificación o a asegurar un resultado acorde con lo deseado.

La definición del problema es un proceso mental que permite especificar con precisión la segunda pregunta por contestar o la meta por lograr. La definición de la estrategia comprende la especificación de un conjunto ordenado de pasos que permiten resolver el problema o lograr la meta planteada; la representación mental es un proceso interno que incluye pasos que permiten comprender el enunciado del problema; la distribución de recursos implica la regulación y uso consciente de los mismos, incluyendo conocimientos, intelecto, etc., disponibles y necesarios para resolver un problema y, finalmente, la supervisión de soluciones introduce el concepto de seguimiento y control como una actividad continua y sistemática que permite verificar el logro de las metas y aplicar los correctivos necesarios durante el proceso, desde el inicio de la planificación hasta la consecución del resultado final o deseado' (p. 34).

En el caso de la investigación aquí presentada, específicamente se estudiaron las habilidades relacionadas con los subprocesos implicados en la resolución de problemas. A saber, estas habilidades se refieren a la definición del problema, la definición de la estrategia para resolver el problema, la representación mental del problema, la distribución de recursos y la supervisión de soluciones.

\section{Problema e hipótesis}

Con base en lo anterior, la presente investigación se orientó en examinar cuáles eran las habilidades cognitivas y metacognitivas que poseían los estudiantes de la población objeto de estudio que ingresaron a cursar el área de ciencias, particularmente bioquímica, y la forma como se podían potenciar estas habilidades a través de estrategias de enseñanza y aprendizaje centradas en la resolución de problemas.

Teniendo en cuenta el problema formulado anteriormente, las hipótesis que se pretendieron contrastar fueron las siguientes:

1. Los estudiantes de la población objeto de estudio, previo a la aplicación de un programa de intervención cognitiva, no han desarrollado las habilidades cognitivas y metacognitivas relacionadas con los procesos directivos para el uso de información, necesarias para la resolución de problemas en el área de las ciencias, particularmente la bioquímica.

2. El empleo de un programa de intervención cognitiva fundamentado en el desarrollo de habilidades cognitivas y metacognitivas relacionadas con los procesos directivos para el uso de información, favorecerá la resolución de problemas en el área de bioquímica.

\section{Diseño metodológico}

El diseño metodológico se dividió en cuatro aspectos: el primero se refiere al tipo de diseño de investigación empleado, el segundo, a la descripción de la población objeto de estudio, el tercero, a las técnicas e instrumentos de recolección de información y, el cuarto, a la estrategia didáctica o programa de intervención cognitiva propuesto para el desarrollo de las habilidades de pensamiento estudiadas. 


\section{1.Diseño de la investigación y población estudiada}

Se empleó un diseño de tipo preexperimental sin grupo control. El grupo experimental estuvo conformado por 12 estudiantes que cursaron bioquímica en el primer semestre del programa de regencia en farmacia en la Corporación Tecnológica de Bogotá, durante el año 2001. El diseño empleó una prueba diagnóstica general (anexo 2) cuyos resultados sirvieron para evaluar las habilidades cognitivas y metacognitivas implicadas en la resolución de problemas.

A partir de los resultados de esta prueba, se diseñó un programa de intervención cognitiva orientado a favorecer el desarrollo de aquellas habilidades cognitivas y metacognitivas en las que se observó que los estudiantes tenían dificultades. La eficacia del programa de intervención se evaluó mediante la aplicación de pruebas postest a medida que se avanzaba en el programa.

\subsection{Técnicas de recolección de la información}

Para la obtención de información, se empleó en todas sus etapas la técnica de observación por encuesta. Al iniciar el programa de intervención, se aplicó a los estudiantes de la población una prueba diagnóstico general (anexo 2) relacionada con una situación problemática, la cual consistió en un problema elaborado por Saugstad y Raaheim (1960, pp. 97-104), quien lo empleó para determinar si las personas establecían subobjetivos en su solución (ver tabla 1). Una vez aplicada la prueba diagnóstico general y analizados los resultados obtenidos en ésta, se inició el programa de intervención durante un curso de bioquímica en el cual se trabajaron cinco situaciones problema, las cuales se describen en la tabla 1.

Tabla 1. Situaciones problema abordadas durante el programa de intervención cognitiva.

\begin{tabular}{|c|c|}
\hline $\begin{array}{l}\text { Situación } \\
\text { problema }\end{array}$ & Problema \\
\hline $\begin{array}{c}\text { PRUEBA } \\
\text { DIAGNÓSTICO }\end{array}$ & $\begin{array}{l}\text { En una de las orillas del río hay tres misioneros y tres caníbales. } \\
\text { Disponen de una barca que puede transportar dos personas a la } \\
\text { vez de una parte a otra del río. El objetivo es transportar a las seis } \\
\text { personas a la otra orilla del río. En ningún momento, el número de } \\
\text { caníbales, en cualquiera de las dos orillas del río, puede exceder al } \\
\text { de misioneros de esa orilla o de lo contrario los caníbales se } \\
\text { comerían a los misioneros. Esta limitación vale solamente cuando } \\
\text { hay al menos un misionero en la del lado del río en la que hay más } \\
\text { caníbales; esto es, vale tener uno, dos o tres caníbales y ningún } \\
\text { misionero en el mismo lado del río porque no habría misioneros } \\
\text { que comer. }\end{array}$ \\
\hline $\begin{array}{l}\text { SITUACIÓN } \\
\text { PROBLEMA No1 }\end{array}$ & $\begin{array}{l}\text { Una empresa de alimentos necesita hacer análisis de cinco } \\
\text { soluciones de igual concentración para verificar si éstas son } \\
\text { carbohidratos y si lo son, determinar cuáles son reductores, } \\
\text { monosacáridos, disacáridos o polisacáridos. Suponga que usted es } \\
\text { el analista de esta empresa, indique la manera cómo haría para } \\
\text { solucionar este problema. }\end{array}$ \\
\hline $\begin{array}{l}\text { SITUACIÓN } \\
\text { PROBLEMA N² }\end{array}$ & $\begin{array}{l}\text { Dada una serie de sustancias, identificar cuáles son lípidos e } \\
\text { indicar cuáles de ellos son insaturados o saturados. }\end{array}$ \\
\hline
\end{tabular}




\begin{tabular}{|l|l|}
\hline SITUACIÓN & $\begin{array}{l}\text { Un paciente lleva una muestra de orina a un laboratorio clínico para } \\
\text { examinar los aminoácidos presentes en dicha muestra, ¿qué } \\
\text { aminoácidos están presentes en la muestra? Suponga que usted el } \\
\text { analista del laboratorio, resuelva el problema, tenga en cuenta que } \\
\text { el análisis solo lo puede hacer por cromatografía en capa fina o en } \\
\text { papel. }\end{array}$ \\
\hline $\begin{array}{l}\text { SITUACIÓN } \\
\text { PROBLEMA N } 4\end{array}$ & $\begin{array}{l}\text { Uno de los alimentos más importantes en la dieta de un ser } \\
\text { humano, sobre todo en los primeros años de vida, es la leche, ya } \\
\text { que aporta gran cantidad de nutrientes al organismo entre ellos } \\
\text { están las proteínas, la lacto albúmina es una de éstas. Determine } \\
\text { experimentalmente el porcentaje de lacto albúmina que está } \\
\text { presente en una muestra de leche, por medio de espectrofoto- } \\
\text { metría. }\end{array}$ \\
\hline PRUEBA POSTEST & $\begin{array}{l}\text { Un estudiante tiene muestras de un tejido vegetal y uno animal y } \\
\text { desea establecer las diferencias estructurales que existen entre las } \\
\text { células de estos dos tejidos utilizando un microscopio. Suponga } \\
\text { que usted es ese estudiante, indique la manera como haría para } \\
\text { resolver dicho problema. }\end{array}$ \\
\hline
\end{tabular}

Las situaciones presentadas a los estudiantes a lo largo del programa de intervención cognitiva, fundamentalmente fueron pruebas de eficiencia en las que, para su solución, era necesario emplear las habilidades cognitivas y metacognitivas relacionadas con los procesos directivos para el uso de información.

Al iniciar cada unidad temática se aplicó a los estudiantes una de las situaciones problema anteriormente descritas, la cual sirvió como prueba pretest sobre las habilidades cognitivas y metacognitivas. Igualmente, al finalizar cada unidad temática, una vez desarrollada la estrategia de intervención, se aplicó la misma situación problema, la cual sirvió como prueba postest. Es decir, en el transcurso del programa de intervención la técnica empleada para evaluar la estrategia consistió en la técnica test-retest.

En las distintas situaciones problema, las cuales fueron cuestionarios de lápiz y papel, se evalúo la habilidad de los estudiantes para definir problemas, seleccionar estrategias, representar problemas, distribuir recursos y supervisar soluciones.

\subsection{Estrategia didáctica o programa de intervención cognitiva}

La estrategia didáctica empleada para el desarrollo de las habilidades en todas las unidades temáticas se fundamentó en las siguientes etapas:

1. Se entregó a cada alumno una guía (anexo 1) donde se explicó cada una de las habilidades cognitivas y metacognitivas relacionadas con la solución de problemas. La guía se analizó, primero, en forma individual y, posteriormente, se discutió y analizó con ayuda de la profesora. La guía fue empleada para analizar, junto con los alumnos, la resolución de la prueba diagnóstica general contestada por cada estudiante.

2. Al iniciar cada unidad temática se entregó a los estudiantes una situación problemática con el fin de que ésta fuera resuelta individualmente extractase, con base en la guía sobre las habilidades cognitivas y metacognitivas entregada y discutida al iniciar el programa. Las situaciones problemáticas planteadas en cada unidad temática, al igual que en la prueba diagnóstica, se refieren a situaciones problema cerradas, con respuesta 
abierta, relacionadas, a excepción de la prueba diagnóstico general, con aspectos conceptuales y experimentales de la bioquímica.

3. En general, en todas las situaciones problemas planteadas a los estudiantes, se les solicitó definir el problema en términos de las discrepancia entre la situación inicial y la situación deseada, las estrategias necesarias para resolver el problema, la representación mental del problema, la distribución de recursos y la supervisión de las soluciones, siguiendo para ello, en todos los casos, un formato como el mostrado en el anexo 3, empleado también en la prueba postest final.

4. Posteriormente, se realizó una plenaria, donde los alumnos plantearon las soluciones del problema, las cuales se sometieron a discusión con orientación de la profesora. La orientación fue gradual y se basó en la formulación de cuestiones que guiaron la reflexión del alumno. Además, se aclararon dudas conceptuales sobre la disciplina y sobre las habilidades necesarias para la resolución del problema, siendo los estudiantes quienes dirigieron el proceso.

5.Una vez realizada la plenaria, los estudiantes llevaron a cabo la solución del problema que ellos propusieron, sin intervención del profesor. Este aspecto implicó trabajo principalmente en el laboratorio.

6.Posteriormente, los estudiantes elaboraron un informe escrito sobre la práctica de laboratorio, el cual fue revisado por La profesora y luego se discutieron los resultados con cada uno de los grupos de estudiantes, quienes debieron corregirlos para entregarlos nuevamente.

7.El informe final que entregaron los estudiantes, contiene los resultados de la solución del problema y una reformulación de cada uno de los pasos propuestos en la prueba diagnóstica que resultaron inadecuados a la luz de la experiencia realizada en el laboratorio.

\section{Resultados y discusión}

\section{1. Resultados prueba diagnóstica general}

Para el análisis de los resultados de la prueba diagnostica general se identificó en las respuestas el empleo de cada una de las habilidades cognitivas relacionadas con los procesos directivos para el uso de información. Las habilidades cognitivas se describen a continuación: (SO).

1. Identificación de la situación inicial

2. Identificación de la situación deseada(SD).

3. Formulación de la discrepancia (DIS).

4.Selección y formulación de la estrategia para la solución de la discrepancia (SE).

5.Representación mental del problema y de la estrategia (RM). 
6.Distribución de los recursos necesarios para la solución del problema (DR).

7.Supervisión de las soluciones, la cual sólo se evaluó en los postest una vez implementada la estrategia de solución (SS).

Debe señalarse que, en la prueba diagnóstica general, como se observa, la situación problema que se planteó a los estudiantes de la población objeto de estudio no se relaciona con los contenidos disciplinares de la bioquímica. Esta característica se debe al hecho de que en la solución de problemas, científicos o no, se emplean las habilidades anteriormente descritas, aunque muchas veces sin que se tenga conciencia de su utilización y sin que dichas habilidades sean formalizadas a través del lenguaje por parte de quienes las emplean.

Así pues, no se empleó una prueba diagnóstica, cuyos contenidos se refirieran a los contenidos disciplinares de la ciencia química, para evitar que los estudiantes dejaran de contestarla por considerar que no tenían los conocimientos disciplinares para resolverla.

Efectivamente, lo que interesa es examinar de qué manera los estudiantes emplean las habilidades cognitivas y metacognitivas en la resolución de una situación problemática, independiente del contexto en el que se utilicen.

La siguiente tabla muestra los resultados obtenidos por los estudiantes de la población en La prueba diagnóstica general según las habilidades analizadas.

Tabla $\mathbf{N}^{0}$ 2. Puntuaciones prueba diagnóstica general según estudiante y habilidad cognitiva y metacognitiva.

\begin{tabular}{|l|l|l|l|l|l|l|l|}
\hline $\begin{array}{c}\mathrm{N}^{\circ} \text { de } \\
\text { estudiante }\end{array}$ & SO & \multicolumn{1}{|c|}{ SD } & DIS & SE & RM & DR & TOTAL \\
\hline 1 & 1 & 1 & 0 & 0 & 1 & 0 & 3 \\
\hline 2 & 1 & 1 & 0 & 0 & 0 & 0 & 2 \\
\hline 3 & 1 & 1 & 0 & 0 & 0 & 0 & 2 \\
\hline 4 & 1 & 1 & 0 & 0 & 0 & 0 & 2 \\
\hline 5 & 1 & 1 & 0 & 0 & 0 & 0 & 2 \\
\hline 6 & 1 & 1 & 0 & 0 & 1 & 0 & 3 \\
\hline 7 & 0 & 0 & 0 & 0 & 0 & 0 & 0 \\
\hline 8 & 1 & 1 & 0 & 0 & 1 & 0 & 3 \\
\hline 9 & 0 & 1 & 0 & 0 & 1 & 0 & 2 \\
\hline 10 & 0 & 1 & 0 & 0 & 0 & 0 & 1 \\
\hline 11 & 1 & 1 & 0 & 0 & 0 & 0 & 2 \\
\hline 12 & 1 & 1 & 0 & 0 & 0 & 0 & 2 \\
\hline TOTALES & 9 & 11 & 0 & 0 & 4 & 0 & 24 \\
\hline
\end{tabular}

1=aprobó

$0=$ no aprobó

Al observar esta tabla, se puede apreciar que todos los estudiantes presentan dificultades en los aspectos necesarios para la resolución de problemas. Se aprecia que en la formulación de la discrepancia, la distribución de recursos y la supervisión de soluciones, los estudiantes obtuvieron puntuaciones de cero, lo que indica que ellos no han potenciado las habilidades cognitivas necesarias para la resolución de problemas. 
En cuanto a las habilidades metacognitivas, se observa que los estudiantes de la población no son conscientes de las habilidades cognitivas que emplean y que son necesarias para la solución del problema. Así pues, los estudiantes no hacen explícito la necesidad de identificar la situación inicial o la situación observada. Adicionalmente, aunque algunos emplean ciertas habilidades, no siempre las emplean de forma organizada, ya que llevan a cabo la representación mental sin formular antes la situación inicial, la observada o la discrepancia, a partir de la lectura comprensiva del enunciado.

\subsection{Resultados postest general}

La tabla 3 muestra los resultados de la prueba final o postest general, la cual se comparó con la prueba diagnóstica general, con el fin de observar la influencia de la estrategia en el mejoramiento de habilidades cognitivas y metacognitivas necesarias en la resolución de problemas una vez desarrollado el programa de intervención cognitiva.

Tabla $\mathbf{N}^{9}$ 3. Puntuaciones postest final según estudiante $y$ habilidad cognitiva $y$ metacognitiva.

\begin{tabular}{|c|c|c|c|c|c|c|c|}
\hline $\begin{array}{c}\mathrm{N}^{\mathrm{O}} \text { de } \\
\text { estudiante }\end{array}$ & SO & SD & DIS & SE & RM & DR & $\begin{array}{c}\text { Puntuación } \\
\text { total }\end{array}$ \\
\hline 1 & 1 & 1 & 1 & 1 & 1 & 1 & 6 \\
\hline 2 & 1 & 1 & 1 & 1 & 1 & 1 & 6 \\
\hline 3 & 1 & 1 & 1 & 1 & 1 & 1 & 6 \\
\hline 4 & 1 & 1 & 0 & 1 & 1 & 1 & 5 \\
\hline 5 & 1 & 1 & 1 & 1 & 1 & 1 & 6 \\
\hline 6 & 1 & 1 & 0 & 1 & 1 & 1 & 5 \\
\hline 7 & 1 & 1 & 1 & 1 & 1 & 1 & 6 \\
\hline 8 & 1 & 1 & 1 & 1 & 1 & 1 & 6 \\
\hline 9 & 1 & 1 & 1 & 1 & 1 & 1 & 6 \\
\hline 10 & 1 & 1 & 1 & 1 & 0 & 0 & 4 \\
\hline 11 & 1 & 1 & 1 & 1 & 1 & 1 & 6 \\
\hline 12 & 1 & 1 & 1 & 1 & 1 & 1 & 6 \\
\hline TOTAL & 12 & 12 & 10 & 12 & 11 & 11 & 68 \\
\hline
\end{tabular}

Al comparar las puntuaciones de la prueba diagnóstica general con el postest final, se observa que Los 12 estudiantes aumentaron las puntuaciones en el postest (ver tabla 4). La comparación de los resultados refleja que el programa de intervención tuvo una gran influencia para mejorar y potenciar aquellas habilidades cognitivas y metacognitivas necesarias para la resolución de problemas.

Tabla $\mathbf{N}^{9} 4$. Comparación prueba diagnóstica general postest final.

\begin{tabular}{|l|c|c|}
\hline & $\mathrm{N}^{\mathrm{O}}$ & $\%$ \\
\hline MEJORARON & 12 & 100,0 \\
\hline IGUALES & 0 & 0,0 \\
\hline DESMEJORARON & 0 & 0,0 \\
\hline
\end{tabular}


Gráfica № 1

Comparación prueba diagnóstica general postest final según estudiante y habilidad cognitiva.

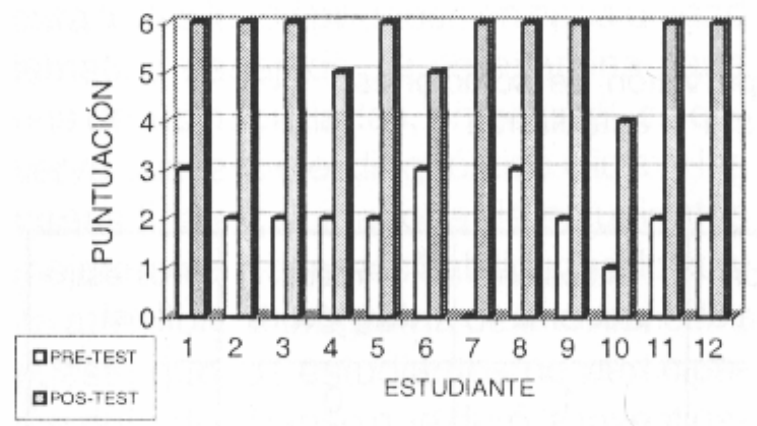

Gráfica № 2

Número de estudiantes que superaron los ítems de las pruebas diagnóstica y postest final.

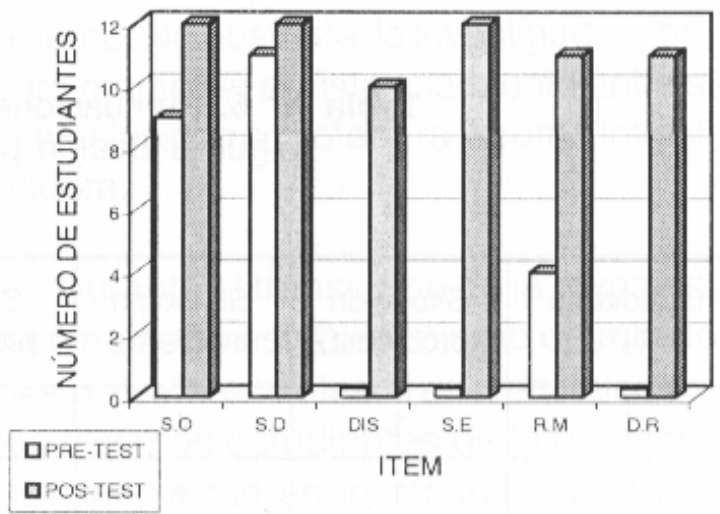

Al observar la gráfica No. 2, se aprecia que el número de estudiantes que superaron los cuatro últimos ítems del postest final, comparados con los de la prueba diagnóstica, aumentó en más del $50 \%$, lo que indica que la estrategia tuvo una gran influencia en la intervención y mejoramiento de las habilidades cognitivas y metacognitivas necesarias para la resolución de problemas.

En cuanto a las habilidades metacognitivas, en general, se observó que a medida que se avanzó en el programa de intervención cognitiva, los estudiantes autónomamente emplearon la heurística general de solución de problemas. Los resultados del postest final comparados con la prueba diagnóstica, muestran una mejora sustancial en la planeación y organización de las habilidades cognitivas necesarias para la solución del problema; así mismo, los estudiantes tienen en cuenta, con base en los resultados de la resolución de las situaciones problema anteriores, las limitaciones en cuanto al manejo, por ejemplo, de los recursos e instrumentos que a nivel de laboratorio se necesitaron para llevar a acabo la estrategia propuesta.

\subsection{Resultados supervisón de soluciones de todas las situaciones problema}

Los resultados que se aprecian en la tabla $\mathrm{N}^{0} 5$ muestran que, tanto en la prueba diagnóstica general, como en las situaciones problema uno y dos, cerca del $50 \%$ de los estudiantes presentan dificultades en la supervisión de soluciones.

En algunos casos, los estudiantes se limitaron a describir o a escribir los resultados obtenidos en la práctica de laboratorio, pero en ningún momento hicieron un análisis de los resultados y de la estrategia utilizada; es decir, no evaluaron si la estrategia que utilizaron fue la adecuada o no.

En la supervisión de soluciones se pretendía que los estudiantes también escribieran las dificultades que se les presentó al aplicar la estrategia y los posibles pasos o caminos que hubieran podido seguir para superarlas, lo que ocurrió en las últimas situaciones problema que se trabajaron, incluyendo el postest final; esto refleja que la estrategia influyó para superar en alguna medida las dificultades que se estaban presentando en este aspecto en las situaciones problema anteriores. 
Tabla $\mathbf{N}^{0}$ 5. Puntuaciones supervisión de soluciones según situación problema y estudiante.

\begin{tabular}{|l|l|l|l|l|l|l|}
\hline Estudiante N & $\begin{array}{l}\text { Situación } \\
\text { problema }\end{array}$ & $\begin{array}{l}\text { Situación } \\
\text { problema }\end{array}$ & $\begin{array}{c}\text { Situación } \\
\text { Problema } \\
3\end{array}$ & $\begin{array}{c}\text { Situación } \\
\text { Problema } \\
4\end{array}$ & $\begin{array}{c}\text { Prueba } \\
\text { Diagnostica }\end{array}$ & $\begin{array}{c}\text { Postest } \\
\text { final }\end{array}$ \\
\hline 1 & 1 & 1 & 1 & 1 & 0 & 1 \\
\hline 2 & 1 & 1 & 1 & 1 & 1 & 1 \\
\hline 3 & 1 & 1 & 1 & 1 & 1 & 1 \\
\hline 4 & 0 & 1 & 1 & 1 & 1 & 1 \\
\hline 5 & 0 & 1 & 1 & 1 & 0 & 1 \\
\hline 6 & 1 & 1 & 1 & 1 & 0 & 1 \\
\hline 7 & 0 & 0 & 1 & 1 & 1 & 1 \\
\hline 8 & 0 & 0 & 1 & 1 & 0 & 1 \\
\hline 9 & 1 & 0 & 1 & 1 & 0 & 1 \\
\hline 10 & 0 & 0 & 1 & 1 & 1 & 1 \\
\hline 11 & 1 & 1 & 1 & 1 & 0 & 1 \\
\hline 12 & 0 & 1 & 1 & 1 & 1 & 1 \\
\hline TOTAL & 6 & 8 & 12 & 12 & 6 & 12 \\
\hline
\end{tabular}

La supervisión de soluciones es una habilidad cognitiva implicada en todo el proceso ejecutivo de solución de un problema y se aplica en todas las fases del manejo de la información, desde el análisis previo de la situación hasta la generación de productos. En este proceso, se requiere de habilidades, tanto metacognitivas como cognitivas, para verificar, controlar y tomar decisiones respecto a todo lo relacionado con la solución del problema.

Al comparar las puntuaciones de la prueba diagnóstica general con el postest final, se observa que los estudiantes, en un 100\%, aumentaron las puntuaciones en el postest final; es decir, que superaron este ítem.

\section{Análisis general de los resultados}

Los resultados descritos anteriormente, se pueden analizar teniendo en cuenta cada una de las habilidades cognitivas y metacognitivas estudiadas y su evolución a través de todo el programa de intervención cognitiva. Así mismo, se pueden analizar los resultados teniendo en cuenta las comparaciones entre el pretest y el postest para todas las habilidades en cada unidad temática trabajada. Si se examina cada una de las habilidades, en general, se observa que, entre el diagnóstico inicial y las tareas finales, la mayoría de estudiantes mejoraron en todas las habilidades, lo cual es atribuible al programa de intervención, puesto que los estudiantes no participaron durante el lapso que duró la investigación en otras actividades similares en las que se procurara el desarrollo de las habilidades estudiadas.

Puesto que la investigación se llevó a cabo en el propio ambiente educativo y no en un laboratorio, sólo se pudo controlar la estrategia o programa de intervención, pero otras variables, como cursos similares a nivel temático que hayan tomado los estudiantes, previa o durante el transcurso de la investigación, no fueron controladas. 
En general, se considera que la actuación de la investigadora estuvo orientada por el marco conceptual que guío la investigación, el cual, adicionalmente, se dio a conocer a los estudiantes de la población objeto de estudio, por lo que los resultados, su tabulación y codificación, se realizaron desde ese marco conceptual aunque pudiesen darse otras formas de análisis e interpretación desde marcos conceptuales distintos.

Por otra parte, se considera que, durante el desarrollo de la investigación, la investigadora es la profesora que orientó el curso de bioquímica, lo que supone una interacción sistemática orientada hacia la consecución de los objetivos del curso y del proyecto. Esta interacción, como profesora y como investigadora, puede ocasionar sesgo en las observaciones, puesto que no hubo la presencia de un observador externo. No obstante, la investigadora procuró mantener el distanciamiento entre su actuación como profesora y como investigadora.

Durante el transcurso de la investigación no hubo establecimiento de relaciones ni preferenciales ni diferenciales con los distintos estudiantes de la población que influyeran en los resultados en las distintas pruebas. Puesto que las pruebas sobre las habilidades cognitivas estudiadas eran pruebas de eficiencia y no de actitud o de valoración, se considera que las respuestas de los estudiantes no fueron influenciadas por aspectos como el respeto social o la aprehensión evaluativa; es decir, por la idea que tuviesen los estudiantes de que estaban siendo juzgados o evaluados por el investigador.

En este mismo sentido, las diferentes pruebas diseñadas evaluaron las habilidades cognitivas y metacognositivas y, por lo tanto, la respuesta de los sujetos a los distintos ítems no dependen de factores como la memoria y el conocimiento disciplinar, sino de las propias habilidades, mediante las cuales el estudiante opera sobre dichos conocimientos; es decir, se considera que mejorar las habilidades que emplean las personas para resolver problemas trae como consecuencia una mejora en los conocimientos sobre dichas habilidades y los conocimientos a los cuales se aplican estas habilidades (conocimiento disciplinar).

Aunque en el diseño de la investigación no se empleó un grupo control, es evidente que los estudiantes del grupo experimental no fueron tratados de una forma especial, más allá del efecto del programa de intervención, por lo que los resultados reflejan el efecto de dicha estrategia y no de una relación preferencial por el grupo experimental.

En cuanto a las variables ligadas al tiempo, como la maduración biológica, es necesario señalar que, durante el lapso que dura la investigación, no se considera que la mejor puntuación obtenida en el postest final, comparada con la prueba diagnóstica general, se deba a dicho factor.

Por otro lado, los mejores resultados obtenidos en las pruebas postest pueden, de hecho, deberse no sólo al programa de intervención, sino al entrenamiento en las aplicaciones sucesivas de los tests, puesto que todos fueron similares y sólo variaba el contenido o temática al cual se aplicaban las habilidades. En este mismo sentido, es posible también que los distintos pruebas empleadas, como pretest y postest, pudiesen predisponer a los estudiantes de la población a mejorar sus actuaciones en el desarrollo de la estrategia.

\section{Conclusiones}


Los resultados obtenidos en la investigación y los análisis descritos previamente, permitieron llegar a las siguientes conclusiones:

1. Los estudiantes de la población objeto de estudio, previo a la implementación del programa de intervención cognitiva, mostraron a través de la prueba diagnóstica, no tener las habilidades cognitivas necesarias para la resolución de problemas, ni tan poco los conocimientos sobre dichas habilidades (habilidades metacognitivas), en el contexto de la bioquímica, lo cual confirma la hipótesis inicial sobre su ausencia en la población.

Las principales dificultades están relacionadas con la definición del problema y la selección y organización de los pasos para la solución del problema. En este caso, se observa que los estudiantes de la población no tienen en cuenta las limitaciones o restricciones planteadas en el enunciado, por lo que la representación mental resulta de alguna forma inadecuada, lo que conduce al planteamiento de estrategias erróneas.

2. El programa de intervención cognitiva diseñado y aplicado a los estudiantes, influyó en el perfeccionamiento, no sólo de las habilidades sino en el conocimiento de dichas habilidades necesarias para la resolución de problemas en el área de la bioquímica, lo cual confirma la segunda hipótesis formulada. En esta investigación, los resultados obtenidos muestran una mejora paulatina de cada una de las habilidades cognitivas implicadas en la resolución de problemas, pues el programa de intervención se constituye en una especie de heurística en donde cada una de las habilidades cognitivas se encuentra relacionada.

En este mismo sentido, el programa de intervención cognitiva provee a los estudiantes de un conjunto de pasos que pueden emplear para plantear una solución probable a la situación problema, obligando al estudiante a pensar sistemáticamente. No obstante, la solución depende también de los contenidos y del dominio de dichos contenidos por parte del estudiante, pues las habilidades operan sobre éstos.

3. Los resultados obtenidos en esta investigación muestran y confirman los fundamentos teóricos sobre los que se sustenta, en el sentido de que las habilidades cognitivas implicadas en la resolución de problemas, e identificadas por diversos autores, son empleadas en muchas ocasiones de forma no consciente por los estudiantes, y es de suponer lo mismo para las personas en general.

Es evidente que las personas se enfrentan frecuentemente a situaciones problemáticas y muchas de ellas son resueltas exitosamente y, posiblemente, otras muchas no, sin que la persona tenga un conocimiento sobre las habilidades que emplea (metacognición); sin embargo, un programa de intervención, como el desarrollado en esta investigación, puede ayudar a aumentar las probabilidades de éxito en la resolución de un problema relacionado no sólo con situaciones escolares sino también con problemas de la vida diaria.

4. Por supuesto, dado que en el diseño de esta investigación no se empleó grupo control, ni observaciones posteriores al impacto de la estrategia, no es posible saber qué tan duradero y extrapolable a otros contextos ha sido el aprendizaje de los estudiantes como consecuencia de su participación en el programa de intervención. De todas formas, es evidente que dichos aprendizajes, como cualquier otro, requieren de un esfuerzo y una práctica sistemática de quien aprende.

5. Los resultados obtenidos son válidos para la población objeto de estudio por cuanto está constituida por adolescentes que han superado ya la educación secundaria y han 
definido, en cierto grado, sus intereses profesionales, lo que supone, además, un nivel de experiencia y madurez cognoscitiva distinta a estudiantes de educación secundaria. Por esta razón, se considera que el programa de intervención fue exitoso en la medida en que se trabajó con personas que estuvieron más dispuestas a participar en el desarrollo del programa.

\section{Bibliografía}

Amnestoy M. (1998) Desarrollo de las habilidades del pensamiento: procesos directivos, ejecutivos y de adquisición de conocimiento. México, Trillas.

Barrows, H. S. y Tablyn, R. M. (1980) Problem-based learning: An aproach to medical education. Citado por: Nickerson, R.; Perkins, D.; Smith, E. (1994) Enseñar a pensar: Aspectos de la aptitud intelectual. $3^{\mathrm{a}}$ ed. Barcelona, Paidós.

Cárdenas S. F. (1998). 'Desarrollo y evaluación de los procesos de razonamiento complejo en ciencias" en Revista TEA. $\mathrm{N}^{0} 3$, pp. 53-68. Bogotá, Universidad Pedagógica Nacional.

Garret, M. R. (1988) "Resolución de problemas y creatividad: implicaciones para el currículo de ciencias", en Revista Enseñanza de las ciencias. Vol. 6, N ${ }^{\circ} 3$, pp. 224-230, Universidad de Barcelona.

Gil, D. y Martínez T. (1988) "La resolución de problemas de física" en: Varela, N. Y Martínez, A. (1997) "Una estrategia de cambio conceptual en la enseñanza de la física: la resolución de problemas como actividad de investigación", en Enseñanza de las ciencias. Vol. 2, $\mathrm{N}^{0} 15$, pp. 173-178, Universidad de Barcelona.

Jessup, M. (1998) Resolución de problemas y enseñanza de las ciencias naturales”, en Revista TEA. N ${ }^{0} 3$, pp. 41-52. Bogotá, Universidad Pedagógica Nacional.

Kempa, R. F. (1986) Resolución de problemas de química y estructura cognoscitiva. Enseñanza de las ciencias. Vol. 4, N² 2, pp. 99-110. Citado por: Jessup, M. "Resolución de problemas y enseñanza de las ciencias naturales", en Revista TEA. $\mathrm{N}^{0} 3$, pp. 41-52. Bogotá, Universidad Pedagógica Nacional.

Miller, G.E.M, (1962) An inquiry in to medical teaching. Citado por: Nickerson, R.; Perkins, D.; Smith, E. (1994) Enseñar a pensar: aspectos de la aptitud intelectual. $3^{\text {a }}$ Ed. Paidós.

Nickerson R.; Perkins, D. y Smith, E. (1994) Enseñar a pensar: aspectos de la aptitud intelectual. Ediciones Paidós. $3^{\mathrm{a}}$ edición.

Novak, J. D. (1988) Teoría y práctica de la educación. Citado por: Jessup, M. (1998) "Resolución de problemas y enseñanza de las ciencias naturales", en Revista TEA. $\mathrm{N}^{0}$ 3, pp. 41-52. Bogotá, Universidad Pedagógica Nacional.

Perales, F. J. (1993) La resolución de problemas: una revisión estructurada. Enseñanza de las ciencias. Vol. 11, $\mathrm{N}^{0}$ 2. Citado por: Jessup C. M. (1998) "Resolución de problemas y enseñanza de las ciencias naturales", en Revista TEA. $N^{0} 3$, pp. 41-52. Bogotá, Universidad Pedagógica Nacional. 
Polyá, G. (1982) Como plantear y resolver problemas. $10^{\mathrm{a}}$ ed. México, Trillas. Citado por: Jessup, M. "Resolución de problemas y enseñanza de las ciencias naturales" en Revista TEA. N ${ }^{0}$ 3, pp. 41-52. Bogotá, Universidad Pedagógica Nacional.

Saugstad, P. y Raaheim (1960) "Problem solving, past experience and availability of functions", en Brithis Journal of Psychology. № 51, pp. 97-104.

Sternberg, R. (1990) "Más allá del cociente intelectual. Una teoría triárquica de la inteligencia humana", Biblioteca de Psicología, Bilbao, Desclée de Brouwer.

\section{Anexo 1}

\section{GUÍA HABILIDADES COGNITIVAS Y METACOGNITÍVAS}

\section{¿Qué es un problema?}

Perales (1993) afirma que "por problema puede entenderse cualquier situación prevista o espontánea que produce, por un lado, un cierto grado de incertidumbre y, por el otro, una conducta tendiente a la búsqueda de su solución" (p. 170).

También se puede afirmar que es un enunciado declarativo o interrogativo acerca de discrepancia para satisfacer una necesidad.

\section{Situación deseada}

- Lo que se quiere que ocurra.

- Lo que debe ocurrir.

- Lo que establecen las normas.

\section{Situación observada}

- Lo que ocurre.

- Lo que se observa en la práctica.

— El resultado de la acción.
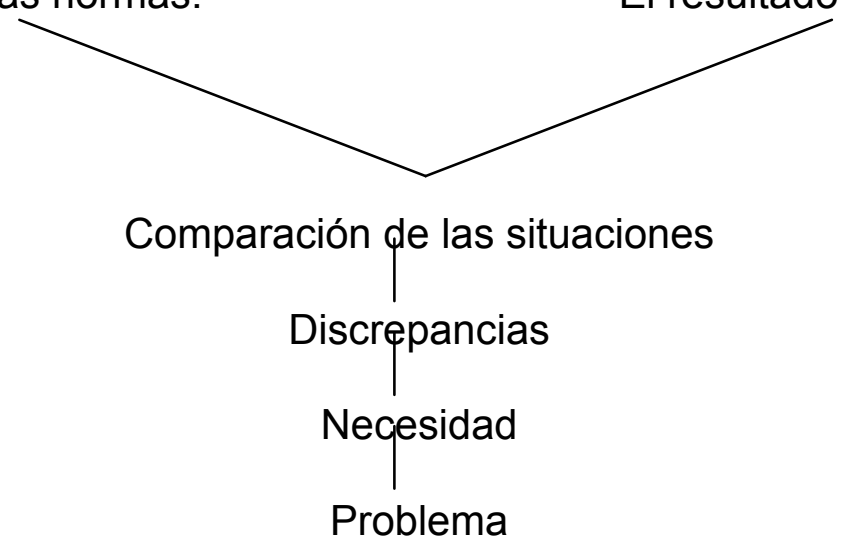

\section{Definición de la estrategia}

Una estrategia es un plan o conjunto de pasos dirigidos a alcanzar un objetivo. En este caso, las secuencias de pasos permiten resolver el problema y tomar ciertos cursos de acción, ya sea para facilitar la toma de decisiones o para investigar la causa que genera el problema.

\section{Actividad}

Coloque en el paréntesis de la izquierda números del 1 al 7 , para indicar el orden en que deben llevarse a cabo dichos pasos para lograr el propósito deseado. 
Estrategia general para resolver un problema:

Seleccionar las alternativas de solución más apropiadas, justificar su factibilidad y analizar las ventajas relativas de los posibles cursos de acción.

( )Definir las limitaciones que impiden la solución del problema.

( )Generar alternativas o cursos de acción que ayuden a resolver el problema.

( )Plantear el propósito general por lograr.

( )Identificar los factores que afectan la situación existente y que limitan el logro de las condiciones deseadas.

( )Analizar la situación existente y la deseada, a fin de explorar y determinar factores, aspectos decisivos, condiciones, limitaciones, oportunidades, etc., que de alguna manera tengan relación con el problema u objetivo por lograr.

( )Considerar la factibilidad de cada alternativa obtenida tomando en cuenta las limitaciones. los recursos, las metas, etcétera.

\section{Representación mental del problema:}

La solución de un problema muchas veces requiere la elaboración de gráficas, dibujo, etc., que ayudan a comprender el significado de lo que se plantea. Este tipo de representación se denomina externa, pues constituye un medio auxiliar que facilita la visualización del problema.

Una vez que se comprende el significado de lo que se plantea en el problema, se adquiere un estado mental que se denomina representación mental o interna del problema. Para lograr dicha representación se requiere realizar procesos de alto nivel cognoscitivo, tales como relaciones, síntesis, interpretaciones, inferencias, etcétera.

\section{Distribución de recursos:}

La distribución de recursos, se aplica a un amplio rango de situaciones, en las cuales se incluyen actividades que requieren el uso de tiempo, energía, esfuerzo, dinero, conocimientos, habilidades, etc. Estos elementos son precisamente los recursos cuya distribución facilita el logro de los objetivos que las personas, aun cuando no estén conscientes, tienen que lograr a cada momento en sus actividades cotidianas.

\section{Supervisión de soluciones}

La supervisión de soluciones es un proceso ejecutivo que se aplica en todas las fases del manejo de la información desde el análisis previo de la situación hasta la generación de productos. Desde el punto de vista administrativo interviene en la planificación, ejecución y control de cualquier actividad y contribuye a mantener un balance entre el resultado de la acción y la intención inicial, a fin de no permitir las desviaciones del proceso como consecuencia del azar, que alejan los productos intermedios o finales que se obtienen de las especificaciones previstas en función del propósito inicial.

La supervisión es un componente fundamental del control de calidad que debe lograr lo siguiente: estar presente en toda actividad desde el momento en que se concibe una idea o un plan hasta que se alcanza la meta planteada; ejercer función regeneradora, esto es, proporcionar la retroalimentación necesaria para ajustar el curso de acción del proceso; propiciar la generación del producto deseado y estar dirigida a lograr resultados óptimos; 
actuar de manera permanente durante todas las fases de planificación después de que se genera el producto, a fin de evaluar sus efectos o su impacto en la población a la cual está dirigido.

\section{Anexo 2}

PRUEBA DIAGNÓSTICO GENERAL

NOMBRE:

FECHA:

SEMESTRE:

En una de las orillas del río hay tres misioneros y tres caníbales. Disponen de una barca que puede transportar dos personas a la vez de una parte a otra del río. El objetivo es transportar a las seis personas a la otra orilla del río. En ningún momento, el número de caníbales en cualquiera de las dos orillas del río puede exceder al de misioneros de esa orilla (o de lo contrario los caníbales se comerían a los misioneros). Esta limitación vale solamente cuando hay al menos un misionero en la del lado del río en la que hay más caníbales; esto es, vale tener uno, dos o tres caníbales y ningún misionero en el mismo lado del río porque no habría misioneros que comer.

En relación con la situación anterior escriba todo lo que usted piensa y proponga para la solución del problema

\section{Anexo 3}

\section{PRUEBA POSTEST FINAL}

NOMBRE:

FECHA:

SEMESTRE:

Un estudiante tiene muestras de un tejido vegetal y uno animal y desea establecer las diferencias estructurales que existen entre las células de estos dos tejidos utilizando un microscopio. Suponga que usted es ese estudiante, indique la manera como haría para resolver dicho problema.

Completar cada uno de los ítem que aparecen a continuación con base en el problema anterior:

\section{DEFINICIÓN DEL PROBLEMA:}


Situación inicial:

Situación deseada:

Discrepancia:

\section{SELECCIÓN DE ESTRATEGIAS}

Paso 1

Paso 2

Paso 3

Paso 4

Paso 5

Paso 6

Paso 7

3. REPRESENTACIÓN MENTAL DEL PROBLEMA

4. DISTRIBUCIÓN DE RECURSOS

\begin{tabular}{|l|l|}
\hline RECURSOS & DESCRIPCIÓN \\
\hline TIEMPO & \\
\hline HUMANOS & \\
\hline ECONÓMICOS & \\
\hline MATERIALES & \\
\hline INTELECTUALES & \\
\hline OTROS & \\
\hline
\end{tabular}

6. SUPERVISIÓN DE SOLUCIONES 